H. Morimoto

Nagoya Math. J.

Vol. 127 (1992), 1-14

\title{
INFINITE DIMENSIONAL CYCLES ASSOCIATED TO OPERATORS
}

\author{
HIROSHI MORIMOTO
}

\section{§ 0. Introduction}

A family of operators defined on infinite dimensional spaces gives rise to interesting cycles (or subvarieties) of infinite dimension which represent a topological or non-topological feature of operator families. In this paper we will give a general theory of these cycles, and give some estimates among them. We will apply this theory, in the final section, to cycles derived from Dirac operators.

We take, as a parameter space of operators, a paracompact space $X$ of infinite dimension. We let $\mathscr{F}=\left\{\mathscr{F}_{x}\right\}_{x \in X}$ be a family of Fredholm operators parametrized by $X$ (this family will be called Fredholm morphism in the sense of ElworthyTromba [5]). Associated to $\mathscr{F}$, there are two kinds of cycles (or subvarieties of $X$ ) that will be our main interest in this paper. One is called solution-cycles, which will be defined as a representation of a global structure of spaces consisting of solutions of $\mathscr{F}$. The other is called index-cycles, which are determined by the index (as a family) of $\mathscr{F}$. In general, the latter cycles can be calculated using the Atiyah-Singer Index Theorem for families of operators. Our aim is to estimate solution-cycles which are not invariant in general by index-cycles which are topologically invariant. In particular, we can prove a non-triviality of solution-cycles for Dirac operators using this estimate. We will denote solution-cycles by $\boldsymbol{\kappa}_{p, q}^{r, s}$, and denote index-cycles by $\phi_{p, q}^{r, s}$. These cycles will turn out to be tied up with a symplectic geometry and a theory of loop groups, which will be mentioned in forthcoming publications (cf. [15]). In the present paper, we will show the exist. ence of cycles, for a family of operators $\mathscr{F}$, satisfying:

$$
|\kappa|_{p, q}^{r, s}(\operatorname{Ker}(\mathscr{F})) \supset|\phi|_{p, q}^{r, s}(\operatorname{Ind}(\mathscr{F})),
$$

here $|*|$ denotes the carrier of a cycle $*$ (or a subvariety) on $X$ (see $\S 2$ ). We will $\overline{\text { Received April }}$ 22, 1991. 
prove, using this estimate, some non-triviality of index-cycles and solution-cycles for Dirac operators in $\S 3$.

Let $E$ and $E^{\prime}$ be infinite dimensional Hilbert spaces (or Kuiper spaces). Let $X$ be a paracompact space (of infinite dimension). We consider a family of Fredholm operators from $E$ into $E^{\prime}$ parametrized by $X$. Let $\eta$ and $\eta^{\prime}$ be infinite dimensional vector bundles over $X$ modelled on $E$ and $E^{\prime}$ respectively, i.e., $\eta, \eta^{\prime}$ have fibres equivalent to $E, E^{\prime}$. A vector bundle morphism $\mathscr{F}: \eta \rightarrow \eta^{\prime}$ will be called a Fredholm morphism if each restriction $\mathscr{F}_{x}$ over $x(\in X)$ is a Fredholm operator. We define numerical index of $\mathscr{F}$ at $x$ by

$$
k_{x}=\operatorname{dim}\left(\operatorname{ker}\left(\mathscr{F}_{x}\right)\right)-\operatorname{dim}\left(\operatorname{coker}\left(\mathscr{F}_{x}\right)\right) .
$$

We will assume that this numerical index is constant with respect to $x \in X$, and denote it by $k$. For simplicity, we suppose $k<0$. (The same argument holds to the case $k>0$.)

Let $p$ and $q$ be non-negative integers with $p-q=-k$. We set,

$$
\chi_{q, p}^{*}(\mathscr{F}) \equiv \chi_{p, q}\left(\mathscr{F}^{*}\right) \equiv\left\{x \in X ; \operatorname{dim}\left(\operatorname{ker}\left(\mathscr{F}_{x}^{*}\right)\right) \geq p\right\} .
$$

These were first investigated by U. Koschorke [7], and later applied to Yang-Mills theory by Atiyah-Jones [2]. We take filtrations of $\eta,\left\{E_{n}\right\}$ and $\left\{E^{\infty-n}\right\}(n=$ $1,2, \ldots)$, consisting of subbundles of $\eta$ such that $E_{1} \subset E_{2} \subset \cdots, E^{\infty-1} \supset E^{\infty-2}$ $\supset \cdots$, and such that $\eta=E_{n} \oplus E^{\infty-n}$ (for any $n$ ).

We now define our solution-cycles as follows. Let $r$ and $s$ be positive integers with $1 \leq r \leq s \leq p$. We set,

$$
\begin{aligned}
& \kappa_{p, q}^{r, s}(\operatorname{Ker}(\mathscr{F}) \\
& \quad=\left\{x \in \chi_{q, p}^{*}(\mathscr{F}) ; \operatorname{dim}\left[\left(\operatorname{ker}\left(\mathscr{F}_{x}\right)\right) \cap E^{\infty-n}\right] \geq \operatorname{dim}\left[\operatorname{ker}\left(\mathscr{F}_{x}\right)\right]-s+r\right\} .
\end{aligned}
$$

This solution-cycle has the following global meaning. Roughly speaking, this cycle can be regarded as a definition of characteristic classes of infinite dimensional bundles defined by kernels of the operators $\left\{\mathscr{F}_{x}\right\}_{x \in \chi_{q, p}^{*}}$ restricted over the set $\chi_{q, p}^{*}$. Precisely speaking, let us consider the restriction of the bundles $\eta, \eta^{\prime}$ and the operators $\mathscr{F}=\left\{\mathscr{F}_{x}\right\}_{x \in \chi_{q, p}^{*}}$ over $\chi_{q, p}^{*}$. We denote the set of all the kernels of $\mathscr{F}$ over $\chi_{q, p}^{*}$ by $K_{p, q}$. For simplicity we assume that $\chi_{q+1, p+1}^{*}=\emptyset$. Then $K_{p, q}$ will become a vector bundle over $\chi_{q, p}^{*}$ of finite constant rank. We denote the $l$-th Chern class of this bundle by $c_{l}\left(K_{p, q}\right)$. Then our solution cycle $\kappa_{p, q}^{r, s}(\operatorname{Ker}(\mathscr{F}))$ corresponds to the following Hankel determinant with coefficient $(-1)^{p q}$ :

$$
\left|\begin{array}{ccc}
c_{p-s+r}\left(K_{p, q}\right) & \cdots & c_{p-s+2 r-l}\left(K_{p, q}\right) \\
\vdots & \ddots & \vdots \\
c_{p-s+1}\left(K_{p, q}\right) & \cdots & c_{p-s+r}\left(K_{p, q}\right)
\end{array}\right| .
$$


This solution cycle is not a topological invariant, and our aim is estimate this cycle by other invariant cycle. We will give such an estimate from below by a cycle denoted by $\phi_{p, q}^{r, s}(\operatorname{Ind}(\mathscr{F}))$ called index-cycle, which is completely determined by the index of the operator family $\mathscr{F}$. This is our main theorem and stated as follows.

THEOREM 1. There exists a cycle $\phi_{p, q}^{r, s}(\operatorname{Ind}(\mathscr{F}))$ such that

$$
|\kappa|_{p, q}^{r, s}(\operatorname{Ker}(\mathscr{F})) \supset|\phi|_{p, q}^{r, s}(\operatorname{Ind}(\mathscr{F})),
$$

and such that the cohomology class corresponding to $\phi_{p, q}^{r, s}(\operatorname{Ind}(\mathscr{F}))$ in $H^{*}(X)$ is a polynomial of Chern classes of the index (as a family) of $\mathscr{F}$, which is calculated as a product of two matrices with coefficient $(-1)^{p q+(p+r)(q-s+r)}$ :

$$
\begin{aligned}
& \left|\begin{array}{ccc}
(-1)^{p} C_{p} & \cdots & (-1)^{p+q+1} C_{p+q+1} \\
\vdots & \ddots & \vdots \\
(-1)^{p+q+1} C_{p-q+1} & \cdots & (-1)^{p} C_{p}
\end{array}\right| \\
& \times\left|\begin{array}{ccc}
(-1)^{p+r} C_{p+r} & \cdots & (-1)^{p+q+s+1} C_{p+q+2 r-s-1} \\
\vdots & \ddots & \vdots \\
(-1)^{p+q+s+1} C_{p-q+s+1} & \cdots & (-1)^{p+r} C_{p+r}
\end{array}\right|
\end{aligned}
$$

here $C_{i}=C_{i}(\operatorname{Ind}(\mathscr{F}))$.

This Main Theorem will be proved in $\S 2$.

The following is an immediate consequence of Theorem 1 :

COROLLARY 1. If the index-cycle $\phi_{p, q}^{r, s}(\operatorname{Ind}(\mathscr{F}))$ is not trivial, then the carrier of the solution-cycle $\kappa_{p, q}^{r, s}(\operatorname{Ker}(\mathscr{F}))$ is not an empty subset of the parameter space $X$.

We will apply this theory to a family of coupled Dirac operators on $S^{4}$ in $\S 3$. Let $D=\left\{D_{[A]}\right\}_{[A] \in \mathscr{A} / \mathscr{G}}$ denote the family of Dirac operators coupled with connections over a fixed $S U(2)$ bundle over $S^{4}$. This family corresponds to an element of the infinite dimensional $K$-theory over $\mathscr{A} / \mathscr{G}$ (the quotient space of the connection space modulo the gauge transformation group with a fixed point). We apply

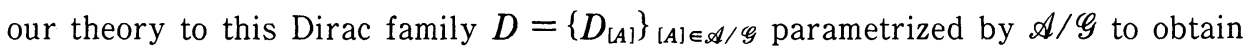
non-triviality of our cycles. In this regard, we have the following. 
THEOREM 2. Let $p$ be an odd prime and $q$ an integer less than $(p-1) / 3$. Then the cohomology class corresponding to the index cycle $\psi_{p-1, q}^{p-1, p-1}(\operatorname{Ind}(D))$ is non-trivial in $H^{*}(\mathscr{A} / \mathscr{G}, \mathbf{Z})$.

As a corollary we have:

COROLLARY 2. The carrier of the solution cycle $\kappa_{p-1, q}^{p-1, p-1}(\operatorname{Ker}(D))$ is not an empty subset of $\mathscr{A} / \mathscr{G}$.

Acknowledgement. The author is very much grateful to Prof. J. Eells and Prof. K.D. Elworthy for their kind hospitality at Mathematics Institute of Warwick University.

\section{§ 1. Infinite dimensional $K$-theory}

This section is devoted to a brief sketch of infinite dimensional $K$-theory. Our reference is K.D. Elworthy and A. Tromba [5]. Throughout this section, $E, E^{\prime}$ will denote infinite dimensional Hilbert spaces (or Kuiper spaces). Let $L_{s p}\left(E, E^{\prime}\right)$ denote the set of all split maps from $E$ int $E^{\prime}$. This space admits a manifold structure (cf. Douady [4]). We denote by $F\left(E, E^{\prime}\right)$ the space of all Fredholm operators from $E$ into $E^{\prime}$, and by $F_{k}\left(E, E^{\prime}\right)$ the set of elements of numerical index $k$. Let $G L(E)$ denote the general linear group of $E$, and let $G L_{c}(E)$ denote the group of automorphisms of $E$ which are of type identity plus compact operator. Let $B F\left(E, E^{\prime}\right)$ be the space of bounded operators of finite rank.

Let $B$ be an arbitrary fixed class of some infinite dimensional spaces. A class of subspaces $P(E, F)$ of $L(E, F)(E, F \in B)$ is called a perturbation class if and only if the followings are satisfied:

(i) If $f, g$ and $h$ are elements of $P(G, E), P(F, G)$ and $P(E, F)$ respectively, then $h \circ f$ belongs to $P(G, F)$ and $g \circ h$ to $P(E, G)$.

(ii) $B F(E, F)+P(E, F)=B F(E, F)$

(iii) $B F(E, F) \subset P(E, F)$.

A typical example of $P\left(E, E^{\prime}\right)$ is the set of all compact operators of $E$ into $E^{\prime}$, and this will be our main object. We denote by $G L_{p}(E)$ a subgroup of $G L(E)$ consisting of operators that are type of Identity plus element of $P(E, E)$. A $G L_{P}(E)$-vector bundle is a vector bundle with fibre $E$ with structure group reduced to $G L_{P}(E)$. Let $X$ and $X^{\prime}$ be paracompact spaces, and consider trivial 
vector bundles $X \times E, X^{\prime} \times E^{\prime}$. Fix some element $T$ of $L\left(E, E^{\prime}\right)$. Then a vector bundle map

$$
f: X \times E \rightarrow X^{\prime} \times E^{\prime}
$$

is called $L(T)$-bundle map if and only if there exist an open covering $\left\{U_{j}\right\}_{j \in J}$ of $X$ and a collection $\left\{E_{j}^{\prime}\right\}_{j \in J}$ of finite dimensional subspaces of $E^{\prime}$ such that for any $x \in U_{j}$, there exists some $h_{x} \in P\left(E, E^{\prime}\right)$ such that

$$
f_{x}=T+h_{x}
$$

and $h_{x}(v) \in E_{j}^{\prime}$ for any $(\mathrm{x}, v) \in U_{j} \times E$. A layer bundle structure on a vector bundle over $X$ modelled on $E$ is defined to be a maximal collection of trivializations:

$$
\begin{gathered}
\left(U_{i}, \phi_{i}\right)_{i \in I}, \\
\phi_{i}: \pi^{-1}\left(U_{i}\right) \rightarrow U_{i} \times E
\end{gathered}
$$

such that each composition:

$$
\phi_{i} \cdot \phi_{j}^{-1}:\left(U_{i} \cap U_{j}\right) \times E \rightarrow\left(U_{i} \cap U_{j}\right) \times E
$$

is an $L(I)$-bundle map (here $I$ denotes Identity). Both the notion of layer bundle morphisms and the notion of equivalence are defined in a natural way among layer bundles.

Let us now consider a continuous family of Fredholm operators. We fix a paracompact space $X$. Let $\pi_{1}: B_{1} \rightarrow X$ and $\pi_{2}: B_{2} \rightarrow X$ be vector bundles over $X$ with fibre $E$ and $E^{\prime}$. A vector bundle morphism

$$
\mathscr{F}: B_{1} \rightarrow B_{2}
$$

is called a Fredholm morphism of index $k$ (or $\mathscr{F}_{k}$-morphism) if and only if each restriction over $x \in X$ of $\mathscr{F}, \mathscr{F}_{x}: \pi_{1}^{-1}(x) \rightarrow \pi_{2}^{-1}(x)$ belongs to $F_{k}\left(E, E^{\prime}\right)$. Layer bundles and Fredholm morphisms are related as follows. (See K.D. Elworthy and A.J. Tromba [5]).

PROPOSITION 1.

(i) Let $\mathscr{F}: B \rightarrow X \times E$ be a $F_{0}$-morphism over a paracompact space $X$. Then there exists a unique layer bundle structure (denoted hereafter by $\{\pi, \mathscr{F}\}_{L}$ ) on $B$ such that $\mathscr{F}$ is an $L(I)$-bundle map.

(ii) Let $\pi_{L}$ be a layer bundle structure on $\pi: B \rightarrow X \times E$. Then there exists a $F_{0}$-morphism $\mathscr{F}: B \rightarrow X \times E$ such that $\pi_{L}=\{\pi, \mathscr{F}\}_{L}$. 
(iii) For $i=1,2$, let $\mathscr{F}_{i}$ be $F_{0}$-morphisms of $\pi_{i}: B_{i} \rightarrow X$ into $X \times E$. Then the corresponding layer bundle structures $\left\{\pi_{i}, \mathscr{F}_{i}\right\}_{L}$ are equivalent to each other if and only if there exists a vector bundle isomorphism $h: B_{1} \rightarrow B_{2}$ such that

$$
F_{2} \cdot h-F_{1}: B_{1} \rightarrow X \times E
$$

is an $L(0)$-bundle map for any trivialization of $B_{1}$.

If we are given a continuous map $\mathscr{F}$ from $X$ into $F_{0}(E, E)$, then this map may be regarded as some $F_{0}$-morphism $\mathscr{F}$ from $B=X \times E$ into $X \times E$. We denote the corresponding layer bundle structure on $\pi: B \rightarrow X$ by $\{\pi, \mathscr{F}\}_{P}$ (notice that Proposion 1 holds also for $P$-bundles). We denote $\{X, \mathscr{F}\}_{P}=\{\pi, \mathscr{F}\}_{P}$. The following proposition is a criterion for the equivalence of layer bundle structures, and follows from Proposition 1.

Proposition 2. For $i=1,2$, let $\mathscr{F}_{i}$ be continuous maps from $X$ into $F_{0}(E, E)$. Then the corresponding layer bundle structures $\left\{X, \mathscr{F}_{i}\right\}_{P}$ are equivalent if and only if there exists a map h from $X$ into $G L(E)$ such that for any $x \in X$,

$$
\mathscr{F}_{1}(x)-\mathscr{F}_{2}(x) h(x) \in P(E) .
$$

Take a complementary filtration of $E,\left\{E_{n}\right\},\left\{E^{\infty-n}\right\}, n=1,2, \ldots$, with $E_{n} \subset$ $E_{n+1}, E^{\infty-n} \supset E^{\infty-n-1}, E_{n} \oplus E^{\infty-n}$ for any $n$. (Note that this filtration is for vector space and is different from filtrations given for bundles such as $\eta$ in the previous section). Let $\iota_{n}: G L(n) \rightarrow G L_{c}(E)$ be natural injections. We set $\iota=\lim _{n \rightarrow \infty} \iota_{n}, G L(\infty)=\lim _{n \rightarrow \infty} G L(n)$. Then we have:

Proposition 3 (K.D. EIworthy, R. Palais). The map $\iota: G L(\infty) \rightarrow G L_{c}(E)$ is a homotopy equivalence.

This proposition holds for any perturbation class $P$ replacing $G L_{c}(E)$ with $G L_{P}(E)$. This result was proved in full generality by R. Palais and K.D. Elworthy independently. For compact operators, it was proved by R. Palais and Svarc. This proposition connects the notions of $G L_{c}$-bundles and $G L(\infty)$-bundles.

Let $k_{c}^{s t}(X)$ denote the group of stable equivalence classes of $G L_{c}$-bundles. Let $[X, B O]$ (resp. $[X, B U]$ ) denote the homotopy classes of maps from $X$ into $B O=\lim B O(n)$ (resp. $B U=\lim B U(n)$ ). The following proposition follows from Proposition 3: 
Proposition 4. $k_{c}^{s t}(X)$ is isomorphic to $[X, B O]$ (resp. $\left.[X, B U]\right)$.

We denote by $k_{F}^{s t}(X)$ the group of stable equivalence classes of $F_{0}$-morphisms over $X$. Then we have the following proposition (cf. Koschorke [7] and Elworthy-Tromba [5]):

Proposition 5. $k_{F}^{s t}(X)$ is isomorphic to $k_{F}^{s t}(X)$.

For $\mathscr{F} \in k_{F}^{s t}(X)$, we will denote the corresponding map by $\Psi[\mathscr{F}] \in[X, B U]$ in view of Proposition 4 and Proposition 5.

\section{$\S 2$. Infinite dimensional cycles}

In this section we will discuss on a structure of cycles derived from kernels of operators, and will derive some estimates for these cycles using index cycles.

Let $E$ be an infinite dimensional Hilbert space (or more generally Kuiper space). From the results of the previous section we have an isomorphism called index:

$$
\text { Ind : }\left[X, F_{0}(E, E)\right] \rightarrow k(X)(=[X, B O](\operatorname{resp} .[X, B U])) .
$$

This yields, through $H^{*}(B O)$ (resp. $H^{*}(B U)$ ), a well-known theory of characteristic classes for families of Fredholm operators (cf. U. Koschorke [7]). We will take hereafter, as a scaler field, complex number field $\mathbf{C}$ and restrict ourselves to complex theory of characteristic classes. In view of the results in the previous section, note that there is also a theory of characteristic classes for the group $k_{F}^{s t}(X)$.

Let $\eta, \eta^{\prime}$ be infinite dimensional vector bundles over $X$, and let

$$
\mathscr{F}: \eta \rightarrow \eta^{\prime}
$$

be a Fredholm morphism, i.e., a continuous family of Fredholm operators parametrized by $X$. Denote the Chern classes of this family by $C(\operatorname{Ind}(\mathscr{F}))$. Let $k$ by the numerical index of $\mathscr{F}$ (here we suppose $k$ is constant). We set, for integers $p, q$ with $p-q=-k$,

$$
\chi_{q, p}^{*}(\mathscr{F})=\chi_{p, q}\left(\mathscr{F}^{*}\right)=\left\{x \in X ; \operatorname{dim}\left(\operatorname{ker}\left(\mathscr{F}_{x}^{*}\right)\right) \geq p\right\} .
$$

We now define kernel-cycles (or solution-cycles) of $\mathscr{F}$. Let $r, s$ be integers with $1 \leq r \leq s \leq p$. Take complementary filtrations of $\eta$ consisting of subbundles $\left\{E_{n}\right\},\left\{E^{\infty-n}\right\}, n=1,2, \ldots$, such that $E_{1} \subset E_{2} \subset \cdots, E^{\infty-1} \supset E^{\infty-2} \supset \cdots$, and $\eta=E_{n} \otimes E^{\infty-n}$ for any $n$. Then we set: 
$\kappa_{p, q}^{\gamma, s}(\operatorname{Ker}(\mathscr{F}))=\left\{x \in \chi_{q, p}^{*}(\mathscr{F}) ; \operatorname{dim}\left[\operatorname{ker}\left(\mathscr{F}_{x}\right) \cap \mathrm{E}^{\infty-s}\right] \geq \operatorname{dim}\left[\operatorname{ker}\left(\mathscr{F}_{x}\right)\right]-s+r\right\}$.

This solution cycle is interesting by itself, but not a topological invariant. There is on the other hand a very useful topologinal invariant, i.e., index of families of operators. Our aim in this section is to estimate solution-cycles from below with cycles (called index-cycles) determined by index of families.

We are now in a position to prove Theorem 1 .

Proof of Theorem 1. We will consider bundle-operators such as identity plus compact operator. In terms of $K$-theory, this situation is described as follows. Take as a perturbation class $P$ the compact operators on the class $B$ consisting of infinite dimensional Kuiper spaces (cf. Section 1). The layer bundle structure is, here, an infinite dimensional vector bundle structure with the structure group reduced to those of $L(I)$-automorphisms that are Identity plus finite rank operator. From K.D. Elworthy and A. Tromba [5] we see that the inclusion map of $G L_{F R}(E)$ into $G L_{P}(E)$ is a homotopy equivalence. Therefore the objects with which we are concerned are vector bundles with the structure group reduced from $G L(E)$ to $G L_{c}(E)$.

Since $\mathscr{F}: \eta \rightarrow \eta^{\prime}$ is a Fredholm morphism and $\eta^{\prime}$ may be assumed to be trivial (as a topological vector bundle), it follows from Proposition 1 that $\mathscr{F}$ induces a layer bundle structure on $\eta$. Although we have restricted ourselves, in the previous section, to the case of numerical index zero, the same argument holds for those of non-zero numerical index.

Let $\pi^{s}: E \rightarrow E^{\infty-s}$ denote the canonical projection. Let $\mathscr{F}^{*}=\left\{\mathscr{F}_{x}^{*}\right\}_{x \in X}$ denote the collection of dual Fredholm morphism of $\mathscr{F}$. Recall that the solution-cycle can be determined (on the subset $\chi_{q, p}^{*}-\chi_{q+1, p+1}^{*}$ ) by the following inequality:

$$
\operatorname{dim}\left[\operatorname{ker}\left(\mathscr{F}_{x}\right) \cap \dot{E}^{\infty-s}\right] \geq p-s+r .
$$

Notice that this is equivalent to inequality:

$$
\operatorname{dim}\left[\operatorname{image}\left(\mathscr{F}_{x}^{*}\right) \cap E_{s}\right] \geq r .
$$

Let $l$ be an arbitrary integer with $0 \leq l \leq p$. Fix $x \in \kappa_{p, q}^{r, s}(\operatorname{Ker}(\mathscr{F}))$, and suppose that

$$
\operatorname{dim}\left[\operatorname{coker}\left(\mathscr{F}_{x}\right)\right]=l .
$$

Then we see that the following equalities hold for this $x$ :

$$
\begin{gathered}
\operatorname{dim}\left[\operatorname{ker}\left(\pi^{s} \cdot \mathscr{F}_{x}^{*}\right)\right]=l+r, \\
\operatorname{dim}\left[\operatorname{coker}\left(\pi^{s} \cdot \mathscr{F}_{x}^{*}\right)\right]=q-s+r+l-p .
\end{gathered}
$$


Consequently we see that $x \in \kappa_{p, q}^{\gamma, s}(\operatorname{Ker}(\mathscr{F}))$ if and only if

$$
x \in \chi_{q, p}^{*}(\mathscr{F}) \cap \chi_{p+r, q-s+r}\left(\pi^{s} \cdot \mathscr{F}^{*}\right) .
$$

Denote the trivial bundle $\mathbf{R}^{p-q} \otimes X$ by $1^{p+q}$. Then there are natural injections :

$$
\begin{gathered}
i: \eta \rightarrow \eta \oplus 1^{p-q}, \\
j: E^{\infty-n} \rightarrow \eta .
\end{gathered}
$$

Consider the Fredholm morphisms $i \cdot j \cdot \pi \cdot \mathscr{F}^{*}$ and $i \cdot \mathscr{F}^{*}$ over $X$. From Proposition 2 , it follows that they give equivalent layer bundle structures. In Section 1 , we noticed that these structures are associated to maps into infinite manifolds of Fredholm operators. We will denote these maps from $X$ into $F_{0}$ by $\Psi[i \cdot j \cdot \pi \cdot \mathscr{F} *]$ and $\Psi\left[i \cdot \mathscr{F}^{*}\right]$ respectively (see the previous section). Notice that they are homotopic. Then we see that $\Psi[j \cdot \pi \cdot \mathscr{F} *]$ and $\Psi[\mathscr{F} *]$ (these are maps of $X$ into $F_{-k}$ ) are homotopic.

Recall that the space $F_{-k}$ (i.e., Fredholm operators of numerical index $-k$ ) is homotopic to $F_{-k+s}$ for any $s$. Therefore there exists a map from $X$ into $F_{-k+s}$ that corresponds to $\Psi\left[\mathscr{F}^{*}\right]$ through this homotopy equivalence. This defines a Fredholm morphism over $X$ that we will denote by $\left(\mathscr{F}^{*}\right)_{+s}$. Denote the corresponding map from $X$ into $F_{-k+s}$ by $\Psi\left[\left(\mathscr{F}^{*}\right)_{+s}\right]$. Similarly, the map $\left(j \cdot \pi \cdot \mathscr{F}^{*}\right)_{+s}$ can be defined.

We see that $\pi \cdot \mathscr{F}$ and $\left(\mathscr{F}^{*}\right)_{+s}$ give the equivalent layer bundle structure, because $\pi \cdot \mathscr{F}$ is equivalent to $\left(j \cdot \pi \cdot \mathscr{F}^{*}\right)_{+s}$. Therefore $\Psi\left[\pi \cdot \mathscr{F}^{*}\right]$ and $\Psi\left[\left(\mathscr{F}^{*}\right)_{+s}\right]$ are homotopic.

Let us consider the following subvariety in $F_{-k+s}$ :

$$
\begin{aligned}
& \mathrm{A}_{p+r, q-s+r} \\
& \quad=\left\{f \in L_{s p}\left(E, E^{\prime}\right) ; \operatorname{dim}[\operatorname{ker}(f)] \geq p+r, \operatorname{dim}[\operatorname{coker}(f)] \geq q-s+r\right\} .
\end{aligned}
$$

This is the closure of the subvariety considered in U. Koschorke [7]. Consider the restrictions of $\pi \cdot \mathscr{F}^{*}$ and $\left(\mathscr{F}^{*}\right)_{+s}$ over $\chi_{q, p}^{*}(\mathscr{F})$. Then we have two sets in $\chi_{q, p}^{*}\left(\mathscr{F}^{*}\right)$ :

$$
\chi_{p+r, q-s+r}\left(\pi \cdot \mathscr{F}^{*}\right), \chi_{p+r, q-s+r}\left(\left(\mathscr{F}^{*}\right)_{+s}\right),
$$

which are exactly the inverse images of the subvariety $A_{p+r, q-s+r}$ through these operators, and which are connected (or cobordant) to each other through the homotopy among $\Psi\left[\pi \cdot \mathscr{F}^{*}\right]$ and $\Psi\left[\left(\mathscr{F}^{*}\right)_{+s}\right]$.

Because it is possible to take the map $\Psi\left[\left(\mathscr{F}^{*}\right)_{+s}\right]$ in general position with respect to the subvariety $A_{p+r, q-s+r}$, we have, taking its inverse image, an 
infinite dimensional cycle in $X$ denoted by $\alpha_{p, q}^{r, s}\left(\left(\mathscr{F}^{*}\right)_{+s}\right)$.

In case that $\chi_{q, p}^{*}(\mathscr{F})$ is in general position, we define:

$$
\varphi_{p, q}^{\gamma, s}\left(\left(\mathscr{F}^{*}\right)_{+s}\right) \equiv \chi_{q, p}^{*}(\mathscr{F}) \cap \alpha_{p, q}^{\gamma, s}\left(\left(\mathscr{F}^{*}\right)_{+s}\right) .
$$

The cycle $\chi_{q, p}^{*}(\mathscr{F})$ represents the following cohomology class of $X$ :

$$
\left|\begin{array}{ccc}
(-1)^{p q} \times \\
(-1)^{p} C_{p}(\operatorname{Ind}(\mathscr{F})) & \cdots & (-1)^{p+q+1} C_{p+q+1}(\operatorname{Ind}(\mathscr{F})) \\
\vdots & \ddots & \vdots \\
(-1)^{p+q+1} C_{p-q+1}(\operatorname{Ind}(\mathscr{F})) & \cdots & (-1)^{p} C_{p}(\operatorname{Ind}(\mathscr{F}))
\end{array}\right| .
$$

It is not difficult to see that the cycle $\alpha_{p, q}^{r, s}\left(\left(\mathscr{F}^{*}\right)_{+s}\right)$ represents the following cohomology class:

$$
\left|\begin{array}{ccc}
(-1)^{(p+q)(q-s+r)} \times \\
(-1)^{p+r} C_{p+r}(\operatorname{Ind}(\mathscr{F})) & \cdots & (-1)^{p+q+s+1} C_{p+q+2 r-s-1}(\operatorname{Ind}(\mathscr{F})) \\
\vdots & \ddots & \vdots \\
(-1)^{p+q+s+1} C_{p-q+s+1}(\operatorname{Ind}(\mathscr{F})) & \cdots & (-1)^{p+r} C_{p+r}(\operatorname{Ind}(\mathscr{F}))
\end{array}\right| .
$$

In case that $\chi_{q, p}^{*}(\mathscr{F})$ is not in general position, we see that there is a cycle in the intersection $\chi_{q, p}^{*}(\mathscr{F}) \cap \alpha_{p, q}^{r, s}\left(\left(\mathscr{F}^{*}\right)_{+s}\right)$ representing the same cohomology class as above. We denote it also by $\varphi_{p, q}^{r, s}\left(\left(\mathscr{F}^{*}\right)_{+s}\right)$.

Because the maps $\Psi\left[\pi \cdot \mathscr{F}^{*}\right]$ and $\Psi\left[\left(\mathscr{F}^{*}\right)_{+s}\right]$ are homotopic, we can find, in the set $\chi_{p+r, q-s+r}\left(\pi \cdot \mathscr{F}^{*}\right)$, a cycle homologous to the cycle $\varphi_{q, p}^{r, s}\left(\left(\mathscr{F}^{*}\right)_{+s}\right)$. We denote it by $\varphi_{q, p}^{r, s}\left(\operatorname{Ind}\left(\mathscr{F}^{*}\right)\right)$. Then it is easily verified that this cycle represents the cohomology class determined by the polynomial in the assertion of Theorem 1 and that

$$
|\kappa|_{p, q}^{r, s}(\operatorname{Ker}(\mathscr{F})) \supset|\varphi|_{p, q}^{r, s}(\operatorname{Ind}(\mathscr{F})),
$$

here $|*|$ denotes the carrier of a cycle $*$.

This completes the proof of Theorem 1 .

Remark. Note that the map $\Psi\left[\left(\mathscr{F}^{*}\right)_{+s}\right]$ is not simply a restriction of $\Psi[\mathscr{F}]$ over $\chi_{q, p}^{*}$. Roughly speaking, the map $\Psi\left[\left(\mathscr{F}^{*}\right)_{+s}\right]$ can be regarded as a Gaussian map of a Gaussian map (or second order Gaussian map) w.r.t. the family of operators $\left(\mathscr{F}^{*}\right)_{+s}$ (cf. [13]). 


\section{§3. Application to Dirac operators}

In this section we apply the results of the previous section to a family of coupled Dirac operators and show a certain non-triviality of solution-cycles and index-cycles for these Dirac operators.

Let $P$ be a principal bundle over $S^{4}$ with the structure group $G=S U(2)$. Fix a point in $S^{4}$, denoted by $\infty$, and fix a base point in the fibre over $\infty$, denoted by $P_{\infty}$. We restrict ourselves to the case of the instanton number $k>0$. Let $\mathscr{G}_{k}$ denote the gauge transformation group that is supposed to be Identity at the base point. Let $\mathscr{A}_{k}$ denote the affine space of all connections $A$ on $P$. We set $\mathscr{C}_{k}=$ $\mathscr{A}_{k} / \mathscr{G}_{k}$.

Let $E_{P}$ denote the associated vector bundle to $P$ (with the standard representation). We consider a family of operators, consisting of Dirac operators coupled to connections $A$ in $P$ acting on negative spinors (with value $E_{P}$ ) taking its value in positive spinors (with value $E_{P}$ ). There is a natural $L^{2}$-extension of coupled Dirac operators (cf. Gromov-Lawson [6]). Henceforth we consider these $L^{2}$-extensions as a family of Dirac oprators. For a given $A \in \mathscr{A}$, we denote by $D_{A}$ the Dirac operator coupled to the connection $A$ which acts on negative spinors with value $E_{P}$. Then this defines a family of operators with parameter space $\mathscr{A}$, and hence gives an element of the infinite dimensional $K$-theory over $\mathscr{C}_{k}=\mathscr{A}_{k} / \mathscr{G}_{k}$ which we will hereafter denote by $D=\left\{D_{[A]}\right\}_{[A] \in \mathscr{C}_{k}}$ (cf. Section 1 ).

We fix a complementary filtration of negative spinors (with value $E_{P}$ ) denoted by $\left\{E_{n}\right\},\left\{E_{\infty-n}\right\}(n=1,2, \ldots)$. Throughout this section $p$ will denote an odd prime number and $q$ will denote a non-negative integer with $q \leq(p-1) / 3$. We shall apply the results of the previous section to the following solution-cycle of Dirac families:

$$
\kappa_{p-1, q}^{p-1, p-1}(\operatorname{Ker}(D))
$$

the carrier of which is the set:

$$
\left\{[A] \in \chi_{q, p-1}^{*}(D) ; \operatorname{ker}\left(D_{[A]}\right) \subset E^{\infty-(p-1)}\right\} .
$$

From Theorem 1, we know that there is an index-cycle $\phi_{p-1, q}^{p-1, p-1}(\operatorname{Ind}(D))$ that estimates the solution-cycle from below, corresponding to the cohomology class ( $\in H^{*}\left(\mathscr{C}_{k}\right)$ ) of the polynomial given in Theorem 1 .

In this section we will prove Theorem 2 . Non-triviality of the cycles $\chi_{q, p}^{*}$ was proved by Atiyah-Jones [2]. For the purpose of the proof of Theorem 2 we must deal with non triviality of polynomials corresponding to subcycles in $\chi_{q, p}^{*}$. 
Proof of Theorem 2. For a positive integer $N$ we denote by $C_{N}\left(\mathbf{R}^{4}\right)$ the qutient space of ordered $N$-tuples of distinct points in $\mathbf{R}^{4}$ by the action of the symmetric group $\Sigma_{N}$, and we will call it the configuration space. For this configuration space, there is a so called t'Hoot construction, which is a map:

$$
\theta_{N}: C_{N}\left(\mathbf{R}^{4}\right) \rightarrow M_{N}
$$

here $M_{N}$ is a manifold of dimension $8 n$ fibred on the moduli space of instantons of degree $N$ with fibre $S O(3)$ (cf. Atiyah-Drinfeld-Hitchen-Mannin [1]). We will denote the standard $N$-dimensional representation of $\pi_{1}\left(C_{N}\left(\mathbf{R}^{4}\right)\right)\left(\cong \sum_{N}\right)$ by $\sigma_{N}$.

We now proceed to prove non-triviality of the cohomology class determined by the polynomial given in Theorem 1 with respect to the cycle $\phi_{p-1, q}^{p-1, p-1}(\operatorname{Ind}(D))$ using a technique of increasing instanton numbers, (the idea of which is due to Atiyah-Jones [2]), we will reduce the problem finally to the vanishing theorem of Lichnerowicz [9]. For this purpose it suffices to prove the non-triviality of the corresponding cohomology class on $\mathscr{A}_{N} / \mathscr{G}_{N}$ for some higher instanton number $N$, for example, $N=3 p q$. Here we use the invariance of $\operatorname{Ind}(D)$ with respect to instanton numbers. Henceforth we will fix $N=3 p q$.

Consider the restriction of $\operatorname{Ind}(D)$ onto the space of self-dual connections of instanton number $N$. It follows from the Lichnerowicz's vanishing theorem that the kernel bundle of $D$ vanishes on the space of self-dual connections. Therefore the problem is reduced to an analysis of the cokernel of $D$, i.e., the kernel bundle of $D^{*}$ on the space of self-dual Yang-Mills connections.

From [1] it follows that the pull-back of this kernel bundle of $D^{*}$ by the map $\theta_{N}$ is isomorphic to a vector bundle on the space $\mathscr{C}_{N}\left(\mathbf{R}^{4}\right)$ that is naturally associated to the representation $\sigma_{N}$. We will denote again this bundle by $\sigma_{N}$, and its Chern classes by $c\left(\sigma_{N}\right)$.

Let $n=3 q$. The $p$-Sylow subgroup of $\sum_{p}$ and $\sum_{n p}$ are $\mathbf{Z}_{p}$ and $\left(\mathbf{Z}_{p}\right)^{n}$ respectively. Let us consider the $p$-primary part of the Chern classes of the vector bundle associated to the standard representation $\sigma_{n p}$. Considering partitions of set $\{1,2, \ldots, n p\}$ into $n$-blocks, we get the following maps:

$$
\begin{aligned}
&\left(C_{p}\right)^{n} \rightarrow C_{n p}, \\
&\left(\sum_{p}\right)^{n} \rightarrow C_{n p} .
\end{aligned}
$$

Note that by restricting Chern class $c\left(\sigma_{n p}\right)$ to $\left(\mathbf{Z}_{p}\right)^{n}$ we have $c\left(\sigma_{p}\right) \ldots c\left(\sigma_{p}\right)$ in $H^{*}\left(\left(\mathbf{Z}_{p}\right)^{n}\right)$, which determines the $p$-primary part of $c\left(\sigma_{n p}\right)$. Let $u$ denote the free generator of $H^{*}\left(\mathbf{Z}_{p}\right)$ of order $p$. Then we see $c\left(\sigma_{p}\right)=1-u^{p-1}$.

The polynomial given by Theorem 1 for the index cycle $\phi_{p-1, q}^{p-1, p}(\operatorname{Ind}(D))$ 
turns out to be the following:

$$
\begin{array}{|}
\left|\begin{array}{ccc}
c_{p-1}(\operatorname{Ind}(D)) & \cdots & (-1)^{q+1} c_{p+q-2}(\operatorname{Ind}(D)) \\
\vdots & \ddots & \vdots \\
(-1)^{q+1} c_{p-q}(\operatorname{Ind}(D)) & \cdots & c_{p-1}(\operatorname{Ind}(D)) \\
& \times & \\
c_{2(p-1)}(\operatorname{Ind}(D)) & \cdots & (-1)^{q+1} c_{2 p+q-3}(\operatorname{Ind}(D)) \\
\vdots & \ddots & \vdots \\
(-1)^{q+1} c_{2 p-q+1}(\operatorname{Ind}(D)) & \cdots & c_{2(p-1)}(\operatorname{Ind}(D))
\end{array}\right|
\end{array}
$$

In $H^{*}\left(\left(\mathbf{Z}_{p}\right)\right)$, we see that the cohomology class associated to this polynomial reduces $(\bmod p)$ to the following:

$$
\left[c_{p-1}\left(\sigma_{n p}\right)\right]^{q} \times\left[c_{2(p-1)}\left(\sigma_{n p}\right)\right]^{q} .
$$

We will see that the coefficient of the term $c_{p-1} \times \cdots \times c_{p-1}$ of this polynomial is the following:

(*) $\left[{ }_{3 q} C_{q} \times{ }_{p-1} P_{q}\right] \times\left[{ }_{2 q} C_{2} \times{ }_{(p-1)-1} P_{2}\right] \times\left[{ }_{2} C_{2} \times{ }_{(p-1)-q-2(q-1)} P_{2}\right]$.

This follows from the observation in $\left[c_{p-1}\left(\sigma_{n p}\right)\right]^{q}$ the contribution of the from (for instance)

$$
\underbrace{c_{p-1} \times \cdots \times c_{p-1}}_{q} \times 1 \times \cdots \times 1
$$

to the term

$$
\underbrace{c_{p-1} \times \cdots \times c_{p-1}}_{3 q}
$$

is ${ }_{3 q} C_{q} \cdot{ }_{p-1} P_{q}$, and the contribution to $\left[c_{2(p-1)}\left(\sigma_{n p}\right)\right]^{q}$ of the form

$$
1 \times \cdots \times 1 \times \underbrace{c_{p-1} \times \cdots \times c_{p-1}}_{2 q}
$$

is

$$
\left.\left.\left.{ }_{2 q} C_{2} \times{ }_{(p-1)-q} P_{2}\right] \times{ }_{2 q-2} C_{2} \times{ }_{(p-1)-q-2} P_{2}\right] \times{ }_{2} C_{2} \times{ }_{(p-1)-q-2(q-1)} P_{2}\right] .
$$

Since $3 q<p$ we see that the integer $(*)$ is not zero $(\bmod p)$. This completes the proof of Theorem 2 . 


\section{REFERENCES}

[1] M.F. Atiyah-V.G. Drinfeld-N.J. Hitchen-Yu.I. Manin, Costruction of instantons, Phys. Lett., 65 A (1978), 185-187.

2 ] M.F. Atiyah-J.D.S. Jones, Topological aspects of Yang-Mills theory, Comm. Math. Phys., 61 (1978), 97-118.

[ 3 ] J.M. Boardman, Singularities of differentiable maps, I.H.E.S. Publ. Math., 33 (1967), $21-57$.

[4] A. Douady, Un espace de Banach dont le groupe linéaire n'est pas connexe, Indag. Math., 68 (1965), 787-789.

[ 5 ] K.D. Elworthy-A. Tromba, Differential structures and Fredholm maps on Banach manifolds, Proc. Sympos. Pure Math., 15 (1970), 95-113.

[6] M. Gromov-H.B. Lawson, Positive scalar curvature and the Dirac operator on complete Riemannian manifolds, I.H.E.S. Publ. Math., 58 (1983), 83-196.

[ 7 ] U. Koschorke, Infinite dimensional $K$-theory and characteristic classes of Fredholm bundle maps, Proc. Sympos. Pure Math., 15 (1970), 95-113.

[ 8 ] N. Kuiper, The homotopy type of the unitary group of Hilbert space, Topology, 3 (1965), 19-30.

[9] A. Lichnerowicz, Spineurs harmoniques, C. R. Acad. Sci. Paris, Ser. A-B, 257 (1963), 7-9.

[10] H. Morimoto, On certain complex analytic cobordism between subvarieties realizing Chern classes of bundles, Nagoya Math. J., 88 (1982), 121-132.

[11] - On the generic existence of holomorphic sections and complex analytic bord. ism, Proc. Japan Acad., 58 Ser. A, No.7(1982), 306-308.

[12] - Realization of Chern classes by subvarieties with certain singularities, Nagoya Math. J., 80 (1980), 49-74.

[13] - Some Morse theoretic aspects of holomorphic vector bundles, Advanced Studies in Pure Math., 3, Geometry of Geodesics and related Topics, North-Holland, (1984), 283-389.

[14] - A theory of infinite dimensional cycles for Dirac operators, Proc. Japan Acad., 65, Ser. A, No. 3(1989), 67-69.

[15] — A Yang-Mills gradient flow and its infinite dimensional limit cycles, Berkeley MSRI (1989), 03924-89.

[16] F. Ronga, Les class duales aux singularités d' ordre deux, Comment. Math. Helv., 47 (1972), 15-35.

[17] R. Thom, Structural stability and morphogenesis, Bonjamin, New York, 1972.

[18] E. C. Zeeman, Catastrophe Theory, selected papers, 1972-1977, Addison-Wesley Publ. Comp. Inc., 1977.

Department of Mathematics

School of Science

Nagoya University

Nagoya 464-01, Japan 